\title{
Die Corona-Krise verschärft Bildungsungleichheit
}

\author{
BETTINA KOHLRAUSCH
}

$\mathrm{D}$ ie Corona-Pandemie hat auch an den Schulen bestehende Ungleichheitsstrukturen offengelegt. Schon vor der Corona-Krise zeichnete sich das deutsche Bildungssystem dadurch aus, dass Bildungschancen stark von der sozialen Herkunft der Kinder und Jugendlichen abhängig waren. Der Zusammenhang zwischen Bildung und sozialer Herkunft ist in Deutschland so stark wie in kaum einem anderen Land der OECD.

Bisher ist noch nicht abschließend empirisch erforscht, welche Folgen die Schulschließungen während der Corona-Pandemie auf die Entwicklung der Bildungsungleichheit bereits hatten und noch haben werden. Erste Befunde sprechen jedoch dafür, dass Schulschließungen und Homeschooling nicht alle Schüler*innengruppen gleich hart treffen; sie verringern vor allem die Bildungschancen von Kindern und Jugendlichen aus den unteren sozialen Schichten. Zwei Mechanismen sind in diesem Zusammenhang besonders relevant:

Erstens beruht das System der Halbtagsschule (selbst in der Grundschule nimmt aktuell nur ca. die Hälfte der Kinder ein Ganztagsangebot wahr) auf der Erwartung, dass ein Teil der Bildungsarbeit von den Eltern bzw. im privaten Umfeld erledigt wird. Diese „Mitarbeit“ können aber nicht alle Eltern gleichermaßen leisten oder an andere Personen, zum Beispiel in Form bezahlter Nachhilfe, delegieren. Entsprechend ist das deutsche Schulsystem nicht in der Lage, ungleiche Voraussetzungen der Kinder und Jugendlichen zu kompensieren. Es tendiert im Gegenteil eher dazu, diese zu verstärken. Dies galt schon vor der Krise, gewinnt in Zeiten des Homeschooling jedoch an zusätzlicher Bedeutung, weil vor allem finanzielle Ressourcen und die damit verbundene Ausstattung mit digitalen Endgeräten oder Wohnraum, der genug Platz zum ungestörten Lernen lässt, ungleich verteilt sind. Gleichzeitig waren Eltern mit geringerem Einkommen und geringerer formaler Bildung auch seltener im Homeoffice und dadurch schlicht nicht vor Ort, um ihre Kinder zu Hause beim Lernen unterstützen zu können. Wenig überraschend empfinden gerade Eltern ohne akademischen Abschluss laut einer aktuellen Studie des Leibniz-Instituts für Wirtschaftsforschung (ifo) die Schulschließungen im Hinblick auf die Lerneinbußen als problematisch und das Lernumfeld zu Hause als unzureichend. Ergänzend dazu fand das Leibniz-Institut für Bildungsverläufe (LIfBi) heraus, dass Eltern mit akademischem Abschluss häufiger der Auffassung waren, dass ihre Fähigkeiten eher ausreichen, um ihre Kinder inhaltlich beim Lernen zu Hause zu unterstützen.

Zweitens trägt die deutsche Schulstruktur, die eine sehr frühe Aufteilung der Schüler*innen auf unterschiedliche Schultypen vorsieht, wesentlich zur Verschärfung von Bildungsungleichheit bei. Dies liegt daran, dass Bildungsentscheidungen der Eltern, aber auch Bildungsempfehlungen des Lehrpersonals, schichtspezifisch getroffen werden. So hatte laut einer Ex- pertise von Klaus Klemm im Auftrag des Deutschen Gewerkschaftsbundes (DGB) ein Grundschulkind von Eltern aus Dienstleistungsberufen bei gleichen Kompetenzen im Jahr 2016 eine 3,37-mal höhere Chance auf eine Gymnasialempfehlung als Kinder aus dem Arbeitermilieu. Kinder aus den unteren sozialen Schichten müssen somit unter ungünstigeren Voraussetzungen mehr leisten, um auf eine höhere Schulform zu kommen. Entscheidend ist, dass diese sehr frühe Aufteilung auf unterschiedliche Schultypen zu einer Schaffung unterschiedlicher Lernumfelder führt. Es kann davon ausgegangen werden, dass die individuellen Lern- und Entwicklungsmöglichkeiten auf einem Gymnasium deutlich besser sind als auf einer Hauptschule. Dies wird zudem durch die äußeren Rahmenbedingungen, wie unterschiedliche Curricula, forciert. Auch während der Schulschließungen herrschten an Gymnasien bessere Bedingungen für das digitale Lernen als an anderen Schultypen. So berichteten vor allem Eltern von Kindern, die ein Gymnasium besuchten, von einem Anstieg interaktiver und/oder rezeptiver virtueller Lernangebote. Auch gibt es nach Angaben der Eltern an Gymnasien häufiger Onlineplattformen, Onlinekurse oder Schul-Clouds. Es liegt vermutlich an der unterschiedlichen Ausstattung und Praxis an den verschiedenen Schultypen, dass Akademikerkinder laut der genannten Ifo-Studie während der Pandemie häufiger in den Genuss von virtuellem Unterricht und individuellen Gesprächen mit Lehrer*innen kamen.

Um Bildungsungleichheiten abzubauen bzw. einer zu erwartenden Verschärfung entgegenzuwirken, ist es notwendig, die beschriebenen Ungleicheitsmechanismen zu adressieren. Das Aktionsprogramm „Aufholen nach Corona für Kinder und Jugendliche“ könnte hierfür ein erster Schritt sein, wenn die eine Milliarde Euro, die in dem „Aufhol-Paket“ für das Nachholen von Lernrückständen vorgesehen ist, genutzt wird, um an den Schulen Strukturen für mehr individuelle Lerngelegenheiten $\mathrm{zu}$ schaffen, die es ermöglichen, Kinder und Jugendliche zielgerichtet besser zu fördern. Die Verzahnung von Förderung und Regelunterricht sollte kommerzieller Nachhilfe vorgezogen werden und ein erster Schritt hin zu einem flächendeckend und qualitativ hochwertigen Ganztagsangebot an allen Schultypen sein.

BETTINA KOHLRAUSCH, Prof. Dr., ist Wissenschaftliche Direktorin des Wirtschafts- und Sozialwissenschaftlichen Instituts (WSI) der Hans-Böckler-Stiftung und Professorin für gesellschaftliche Transformation und Digitalisierung an der Universität Paderborn. Arbeitsschwerpunkte: Bildungsungleichheit, soziale Ungleichheit, Effekte der Digitalisierung auf Arbeit und Qualifizierung, Wandel politischer Einstellungen.

bettina-kohlrausch@boeckler.de 